Journal of Nonlinear Mathematical Physics

\title{
Particle paths in Stokes' edge wave
}

Raphael Stuhlmeier

To cite this article: Raphael Stuhlmeier (2015) Particle paths in Stokes' edge wave, Journal of Nonlinear Mathematical Physics 22:4, 507-515, DOI:

https://doi.org/10.1080/14029251.2015.1113048

To link to this article: https://doi.org/10.1080/14029251.2015.1113048

Published online: 04 January 2021 


\title{
Particle paths in Stokes' edge wave
}

\author{
Raphael Stuhlmeier \\ Faculty of Civil \& Environmental Engineering \\ Technion - Israel Institute of Technology \\ Haifa, 32000, Israel \\ raphaels@technion.ac.il
}

Received 24 August 2015

Accepted 5 October 2015

\begin{abstract}
We treat the particle motion in Stokes' linear edge wave along a uniformly sloping beach. By a rotation of the coordinate frame, we show that there is no particle motion in the direction orthogonal to the sloping beach, and conclude that particles have a longshore drift in the direction of wave propagation which decreases with depth and distance from the shoreline. We discuss the application of this rotated coordinate frame to higher mode (Ursell) and weakly nonlinear (Whitham) edge waves, and show that the weakly nonlinear case is identical to that for two-dimensional deep-water Stokes waves.
\end{abstract}

Keywords: Edge waves; particle trajectories; deep-water waves.

2010 Mathematics Subject Classification: 76B15, 76B07

\section{Introduction}

The matter of particle motion beneath water waves is one of the most natural of questions; it is immediately clear from observations that water particles do not generally travel with the speed of the waveform, but the exact character of their motion in different types of waves has been the matter of much investigation. In particular, considerable progress has been made in the past decade, with the elucidation of the particle paths beneath irrotational, periodic (Stokes) waves by Constantin [1] and those beneath irrotational, solitary waves by Constantin and Escher [2]. These theoretical results have been recently confirmed both numerically [3,4] as well as experimentally [5].

Mathematical investigations of particle trajectories in linear, irrotational water waves go back to G. Green and G. B. Airy in the mid $18^{\text {th }}$ century (see [6]). These authors proceeded from a linearization of the governing equations to an investigation of plane, periodic waves on water of infinite and finite depth, respectively. Treating the differential equations describing the particle paths by a further linearization about the initial position, they found the now familiar result that particle paths in deep water are closed circles with a radius exponentially decreasing with depth, while in finite depth particle paths are ellipses which flatten out as they approach the bottom. This contrasts with the results of the exact, irrotational theory in water of infinite [7] or finite depth [1], where no closed particle paths exist. Note, however, that the presence of even a uniform underlying current might lead to the formation of closed particle paths at a certain depth [8].

Rather than circumvent the difficulties of the linear problem in finite depth by introducing a second linearization - as Airy had done in 1841 - Constantin and Villari [9] undertook to study the particle trajectories directly, via a phase-plane analysis of the two nonlinear ordinary differential equations. Soon thereafter, Constantin, Ehrnström and Villari [10] revisited the problem for infinite 
depth - initially treated by Green in 1839 - again without employing a second linearization. In both cases, the results were striking: there are no closed orbits for water particles in the framework of the linear, irrotational theory. Indeed, in stark contrast to Airy and Green's classical results, which may be found in nearly any textbook on water waves, the particle paths of the linear theory exhibit a forward drift in the direction of propagation.

While particle trajectories in planar water waves have been studied extensively, making use of the powerful tools from complex analysis and the phase plane available there, the absence of these techniques for the full three dimensional problem means that there has been a dearth of progress in this case. We take up the question raised by Okamoto and Shoji [11] on the particle paths of a fairly accessible, but truly three dimensional, wave - the edge wave on a sloping beach.

First discovered by Stokes in 1846, this trapped wave, which moves along the beach (the longshore direction) and has an amplitude decaying with distance from the shoreline was long regarded as a mathematical curiosity. Only in the mid $20^{\text {th }}$ century was the edge wave implicated in the formation of numerous coastal features, from beach cusps to crescentic bars, and were measurements of edge wave activity in the nearshore undertaken (see Johnson [12] and references therein).

In what is to follow, we shall first outline the governing equations and present the key features of Stokes' edge wave, a linear, irrotational wave on a sloping bed. In light of the intractability of the equations for the particle paths in this case, and taking a cue from the remarkable commonalities between Gerstner's exact, rotational wave and the classical linear, irrotational theory, we derive Stokes' edge wave in a rotated coordinate frame and show that the velocity field therein is wholly two-dimensional. It is then a simple matter to apply the theorem of Constantin, Ehrnström and Villari to show that the particle paths are non-closed and exhibit a forward drift in the direction of wave propagation. In a subsequent discussion, we use this rotated coordinate frame to investigate other edge waves. In particular, while Ursell's higher mode edge waves ${ }^{\mathrm{a}}$ exhibit an essential cross-shore variation, the weakly-nonlinear theory is shown to reduce to a two-dimensional problem identical to that of higher-order deep-water Stokes theory.

\section{Mathematical Preliminaries}

As our point of departure, the governing equations in a coordinate frame with $z$ pointing vertically upwards are written in dimensional variables as follows:

$$
\begin{aligned}
& \frac{D u}{D t}=-P_{x} \\
& \frac{D v}{D t}=-P_{y} \\
& \frac{D w}{D t}=-P_{z}-g \\
& u_{x}+v_{y}+w_{z}=0 \\
& P=P_{\text {atm }} \text { on the water surface } z=\eta(x, y, t) \\
& w=\frac{D \eta}{D t} \text { on the water surface } z=\eta(x, y, t) \\
& w=\frac{D b}{D t} \text { on the stationary bed } z=b(x, y)
\end{aligned}
$$

${ }^{\mathrm{a}}$ There is some mystery concerning the derivation of this solution, see the remarks by Whitham [13, Sec. 7.5]. A systematic derivation thereof and illuminating discussion may be found in Ehrenmark [14]. 
Here $(u, v, w)$ is the fluid velocity field, $g$ the acceleration of gravity, $P$ the pressure, $P_{\text {atm }}$ a constant denoting atmospheric pressure, $\eta$ the free water surface and $b$ the rigid, impermeable bed. In order to proceed with a linearization, we choose to measure the pressure relative to hydrostatic pressure via the introduction of a new pressure variable $p$ such that

$$
P=P_{a t m}+g z+g p .
$$

Therewith, the dynamic boundary condition (2.1e) takes the more amenable form

$$
P=\eta \text { on the water surface } z=\eta(x, y, t) \text {. }
$$

Scaling via a small parameter such that $(\mathbf{u}, p, \eta) \rightarrow \varepsilon(\mathbf{u}, p, \eta)$, one arrives at the linear system

$$
\begin{aligned}
& u_{t}=-p_{x} \\
& v_{t}=-p_{y} \\
& w_{t}=-p_{z} \\
& u_{x}+v_{y}+w_{z}=0 \\
& p=\eta \text { on the water surface } z=0 \\
& w=\eta_{t} \text { on the water surface } z=0 \\
& w=u b_{x}+v b_{y} \text { on the bed } z=b(x, y)
\end{aligned}
$$

for which we assume a uniformly sloping bed specified by

$$
b(x, y)=-x \tan \alpha,
$$

yielding Stokes' edge wave when we make an ansatz for harmonic waves traveling in the longshore (y) direction

$$
\varphi(x, y, z, t)=A \exp (i(k y-\omega t)+l z-m x) .
$$

The resulting algebraic equations then yield a dispersion relation $\omega=\sqrt{k \sin \alpha}$ and the potential

$$
\varphi(x, y, z, t)=A \exp (i(k y-\sqrt{k \sin \alpha} t)+z k \sin \alpha-x k \cos \alpha) .
$$

From this, we can see that the wave motion decays both as one moves away from the shoreline (in the $x$ direction) and as one descends into the fluid (in the $z$ direction). (More details of this derivation may be found in Johnson [12].) Unfortunately, the system of nonlinear ordinary differential equations which describe the particle paths

$$
\begin{aligned}
& \dot{x}=-k \cos \alpha \varphi(x, y, z, t) \\
& \dot{y}=i k \varphi(x, y, z, t) \\
& \dot{z}=k \sin \alpha \varphi(x, y, z, t)
\end{aligned}
$$

is essentially intractable. We are confronted with a truly three dimensional wave, and despite the considerable simplifications attendant to our linearization, it seems a knowledge of the particle motions beneath Stokes' edge wave is out of reach. 


\section{Analogies and coordinates}

Our further progress will be illuminated by making brief note of the analogies between linear, deep-water theory and the exact, rotational wave developed by Franz Josef Gerstner in 1804. This wave is the only known exact solution to the governing equations in water of infinite depth; it features circular particle paths whose radius decreases with depth, and consequently a trochoidal wave profile which propagates with a celerity $c=\sqrt{g / k}$, precisely the linear deep-water wave speed. Gerstner's wave was modified by Constantin in 2001 [15] to describe an explicit, exact edge wave solution, albeit in Lagrangian coordinates - a solution which surprisingly shares the dispersion relation of the Stokesian edge wave.

For our purposes, the main feature of interest in Constantin's trochoidal edge wave is the decomposition of the particle trajectories. The solution is given in terms of the Lagrangian labels $a, b$ and $c$ - and it is presented in a coordinate frame rotated so that the $x$ direction is along the sloping bed, and the $z$ coordinate is orthogonal to it - i.e. a coordinate frame rotated with respect to the usual one by an angle $\alpha$ (see Fig. 1).
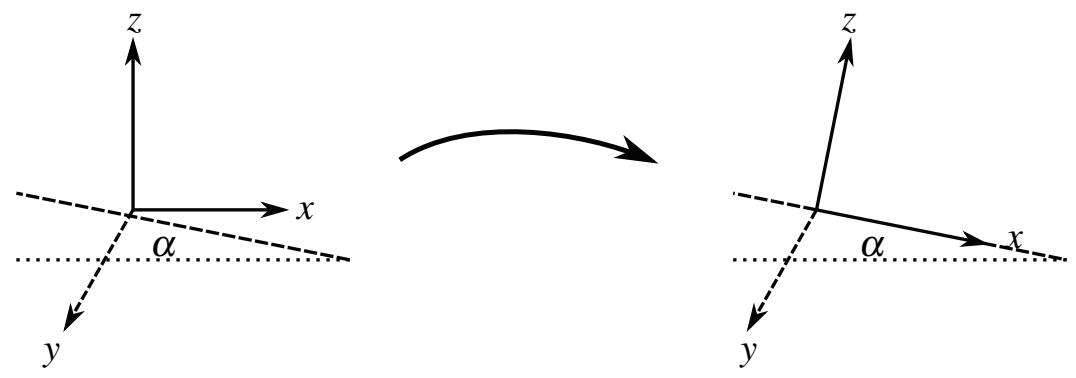

Fig. 1. Rotation of coordinate axes.

In this Gerstner edge wave, it is immediately seen that the component of the velocity orthogonal to the sloping bed ( $w$ in the new coordinates) is zero. Indeed, the structure of the solution is such that in each plane of constant $z$, the motion is described by a planar Gerstner wave. This invites the possibility - in light of the many other commonalities between Gerstner and linear theory - that the same choice of coordinates might allow us to progress on the particle paths in Stokes' edge wave.

To this end, let us reconsider the dimensional system (2.1a)-(2.1g). In a coordinate frame rotated clockwise by an angle $\alpha$, such that $x$ points downward along the sloping bed and $z$ is orthogonal thereto, the force of gravity

$$
(0,0,-g) \text { becomes }(g \sin \alpha, 0,-g \cos \alpha) \text {. }
$$

The kinematic variables are independent of orientation, and the impermeability condition on the bed simplifies considerably, yielding 


$$
\begin{aligned}
& \frac{D u}{D t}=-P_{x}+g \sin \alpha \\
& \frac{D v}{D t}=-P_{y} \\
& \frac{D w}{D t}=-P_{z}-g \cos \alpha \\
& u_{x}+v_{y}+w_{z}=0 \\
& P=P_{a t m} \text { on the water surface } z=\eta(x, y, t) \\
& w=\frac{D \eta}{D t} \text { on the water surface } z=\eta(x, y, t) \\
& w=0 \text { on the bed } z=0
\end{aligned}
$$

To find the correct linearization, we again introduce a new pressure variable $p$ measuring deviations from hydrostatic pressure, such that

$$
P(x, y, z, t)=P_{a t m}+g x \sin \alpha-g z \cos \alpha+g p(x, y, z, t) .
$$

We also write the free surface as a perturbation of the flat surface, that is

$$
\eta(x, y, t) \text { is replaced with } x \tan \alpha+\eta(x, y, t) \text {. }
$$

Assuming that our variables are suitably nondimensionalized (a feat which can be easily accomplished through a more careful introduction of various parameters, such as typical wavelength $\lambda$, water depth $h_{0}$, amplitude $a$, and would result in the appearance of the usual amplitude parameter $\varepsilon=a / h_{0}$ and the shallowness parameter $\delta=h_{0} / \lambda$ ) the equations take the form

$$
\begin{aligned}
& \frac{D u}{D t}=-p_{x} \\
& \frac{D v}{D t}=-p_{y} \\
& \frac{D w}{D t}=-p_{z} \\
& u_{x}+v_{y}+w_{z}=0 \\
& p=\eta \cos \alpha \text { on } z=x \tan \alpha+\eta \\
& w=u \tan \alpha+\eta_{t}+u \eta_{x}+v \eta_{y} \text { on } z=x \tan \alpha+\eta \\
& w=0 \text { on the bed } z=0
\end{aligned}
$$


The corresponding linear system is then simply

$$
\begin{aligned}
& u_{t}=-p_{x} \\
& v_{t}=-p_{y} \\
& w_{t}=-p_{z} \\
& u_{x}+v_{y}+w_{z}=0 \\
& p=\eta \cos \alpha \text { on the water surface } z=x \tan \alpha \\
& w=u \tan \alpha+\eta_{t} \text { on the water surface } z=x \tan \alpha \\
& w=0 \text { on the bed } z=0
\end{aligned}
$$

As we assume the flow field to be irrotational, we make again the harmonic wave ansatz for longshore propagating waves:

$$
\varphi(x, y, z, t)=A \exp (i(k y-\omega t)+l z-m x)
$$

The kinematic boundary condition on the bed $(3.5 \mathrm{~g})$ immediately implies that the fluid velocity component normal to the bed vanishes. The Stokes' edge wave in these coordinates is described by

$$
\begin{aligned}
& \varphi(x, y, z, t)=A \exp (i(k y-\sqrt{k \sin \alpha} t)-k x), \\
& \eta(x, y, t)=i \omega \sec \alpha A \exp (i(k y-\sqrt{k \sin \alpha} t)-k x) .
\end{aligned}
$$

This is seen to describe a wave propagating in the longshore direction with $\omega=\sqrt{k \sin \alpha}$, whose motion dies out exponentially as one moves away from the shoreline and as one descends into the fluid, cf. (2.4). In fact, while (3.6) can be found by solely rotating (2.4) (see Yeh [16]), this does not present the whole story. In particular, it does not enable a subsequent investigation of nonlinear edge waves (see Discussion below).

Consequently, we see that particle trajectories are the solutions to

$$
\begin{aligned}
& \dot{x}=-k A e^{-k x} \cos (k y-\sqrt{k \sin \alpha} t), \\
& \dot{y}=-k A e^{-k x} \sin (k y-\sqrt{k \sin \alpha} t), \\
& \dot{z}=0 .
\end{aligned}
$$

Theorem 1. The motion of the fluid particles is entirely confined to planes parallel to the sloping bed, and each particle moves in a non-closed trajectory, exhibiting a forward drift in the direction of wave motion. This forward drift decreases with $x \rightarrow \infty$.

Proof. See the proofs of Theorem 3.2 and Corollary 3.4 of [10].

\section{Discussion}

The foregoing analysis has been remarkably simple, and raises the natural question whether a rotation of coordinates might be useful in elucidating interesting properties of other edge waves. Within the confines of purely linear theory, described by (3.5a)-(3.5g), the ansatz adopted by Ursell [17] 
and Roseau [18] (once again, see the review by Johnson [12])

$$
\begin{aligned}
& \phi(x, y, z, t)=A(x, z) e^{i(k y-\omega t)}, \\
& p(x, y, z, t)=i \omega A(x, z) e^{i(k y-\omega t)}, \\
& \eta(x, y, t)=i \omega \sec \alpha A(x, z) e^{i(k y-\omega t)},
\end{aligned}
$$

leads us to the system for $A(x, z)$ :

$$
\begin{aligned}
& A_{x x}+A_{z z}-k^{2} A=0 \\
& A_{z}=\tan \alpha A_{x}+\omega^{2} \sec \alpha A \text { on } z=x \tan \alpha \\
& A_{z}=0 \text { on } z=0
\end{aligned}
$$

While we might be initially optimistic at recovering the zero-mode solution of Ursell

$$
A(x, z)=A_{0} e^{-k x},
$$

it quickly becomes apparent that the higher modes cannot reduce to superpositions of planar waves. Assuming $z$-independence in the surface boundary condition and integrating shows that the zeromode with $k=\omega^{2} \csc \alpha$ is the only such solution. Indeed, Ursell's next higher mode (for beach angles $\alpha \leq \pi / 6$ ) may be written

$$
A(x, z)=A_{0} e^{-x k}-A_{0} \frac{\tan \alpha}{\tan 2 \alpha} e^{-k x \cos 2 \alpha}\left(e^{-k z \sin 2 \alpha}+e^{k z \sin 2 \alpha}\right),
$$

demonstrating a cross-shore variation. (Ursell's general solution in rotated coordinates may be found in Mok and Yeh [19], who employ the same without commenting on the matter of particle paths.)

The next natural question is whether nonlinear edge waves might reduce to a two-dimensional motion in our $(x, y)$-plane. This might be asked of both the weakly-nonlinear edge waves due to Whitham [20], or - in light of successes in studying particle paths in the fully nonlinear Stokes' wave in deep water by Henry [7] - for the full, nonlinear theory. Note that Henry's result, like that of Constantin [1] which preceded it, depends crucially on two-dimensionality. It thus seems likely that a result on particle motion in nonlinear edge waves will be possible if and only if the motion can be shown to be two-dimensional.

We shall address only the problem of weakly-nonlinear edge waves, and leave the fully nonlinear case as a topic for future study. Once again, our analysis will bear out the fact that a rotation of the coordinate frame seems to be the proper lens through which to view the edge wave problem. To facilitate our expansion, we recast equations $(3.4 \mathrm{a})-(3.4 \mathrm{~g})$ in the more convenient potential form under the assumption of irrotationality, and make an ansatz for longshore traveling waves $\phi(x, y, z, t)=\phi(x, y-c t, z)$, leading to the system:

$$
\begin{aligned}
& \Delta \phi=0, \\
& c \phi_{y}-\frac{1}{2}(\nabla \phi)^{2}-\eta \cos \alpha=0 \text { on } z=x \tan \alpha+\eta, \\
& \phi_{z}=\phi_{x} \tan \alpha-c \eta_{y}+\phi_{x} \eta_{x}+\phi_{y} \eta_{y} \text { on } z=x \tan \alpha+\eta, \\
& \phi_{z}=0 \text { on } z=0 .
\end{aligned}
$$

Expanding $\phi \sim \varepsilon \phi_{1}+\varepsilon^{2} \phi_{2}+\varepsilon^{2} \phi_{3}, \eta \sim \varepsilon \eta_{1}+\varepsilon^{2} \eta_{2}+\varepsilon^{2} \eta_{3}$, and $c \sim c_{0}+\varepsilon c_{1}+\varepsilon^{2} c_{2}$, we find that the solutions to our suitably rotated equations are at each order independent of $z$ and identical to 
the deep-water Stokes theory, save for dependence on the angle $\alpha$. Defining an offshore coordinate $x^{\prime}:=x \sec \alpha$ on the water surface $z=x \tan \alpha$, and $A:=e^{-k x^{\prime} \cos \alpha}$, we find correct to third order:

$$
\begin{aligned}
\phi & \sim \varepsilon \frac{c_{0}}{k} \sin (k y) e^{-k x} \\
\eta & \sim \frac{\varepsilon A \tan \alpha}{k}\left(\cos (k y)+\frac{1}{2} \varepsilon A \cos (2 k y)+\frac{1}{8} \varepsilon^{2} A^{2} \cos (k y)+\frac{3}{8} \varepsilon^{2} A^{2} \cos (3 k y)\right) \\
\omega & \sim \sqrt{k \sin \alpha}\left(1+\frac{1}{2} \varepsilon^{2} A^{2}\right)
\end{aligned}
$$

The details of the calculation are essentially analogous to those found in Wehausen and Laitone [21, Sect. 27], reproducing the amplitude-dependent dispersion relation also found by Whitham [20]. This identity between the dispersion relation of Whitham and deep-water Stokes theory was also noted by Yeh [16,22], albeit without providing further calculations.

\section{Conclusions}

We have shown that the flow field of a truly three-dimensional wave - Stokes' edge wave - reduces with proper choice of coordinates to the motion of linear plane waves. The fact that such a transformation might bear fruit was indicated by the theory of Gerstner's waves, where the same coordinate frame allowed for the construction of exact edge waves.

This particle motion, as described by the system (3.8a)-(3.8c), may either be treated by a further linearization about the initial position, leading to the classical result that the particles move in closed circles whose radius decreases with depth and distance from shore, or may be analyzed directly via phase plane methods. This latter analysis, an application of recent results of Constantin, Ehrnström and Villari [10], yields that the fluid particles have a drift in the direction of wave propagation, along the shoreline, though this drift decreases as one descends into the fluid and as one moves away from the beach.

While Ehrnström et al [23] have made note of the resemblance between the edge waves found in the small amplitude limit for a small, slowly-varying beach slope (see Johnson [24] for the derivation) and linear theory, the results above go a step further in demonstrating, without restriction on the beach slope, that edge waves and deep-water waves are in fact connected by a simple rotation of the coordinate system. Using this same rotated coordinate frame, the higher-mode theory of edge waves is found to reduce exactly to deep-water Stokes' waves, a considerable simplification compared to Whitham's derivations [20].

Our systematic derivation of the equations, and subsequent demonstration for both linear and weakly-nonlinear theory that the edge waves found therein are two-dimensional, with motion confined to planes of constant $z$ in the new coordinates, partially answers the question posed by Okamoto and Shoji [11] on the nature of particle paths in edge waves. The related matter of particle trajectories for fully nonlinear edge waves remains an interesting open problem.

\section{Acknowledgements}

The author was supported in part at the Technion by a Fellowship from the Lady Davis Foundation. The author also acknowledges support from Israel Science Foundation grant 464/13.

\section{References}

[1] A. Constantin, The trajectories of particles in Stokes waves, Inven. Math. 166(3) (2006) 523-535. 
[2] A. Constantin and J. Escher, Particle trajectories in solitary water waves, Bull. Amer. Math. Soc. 44(3) (2007) 423-431.

[3] Y.-Y. Chen, H.-C. Hsu, and G.-Y. Chen, Lagrangian experiment and solution for irrotational finiteamplitude progressive gravity waves at uniform depth, Fluid Dyn. Res. 42(4) (2010) 045511.

[4] D. Clamond, Note on the velocity and related fields of steady irrotational two-dimensional surface gravity waves, Philos. Trans. R. Soc. A Math. Phys. Eng. Sci. 370(1964) (2012) 1572-1586.

[5] M. Umeyama, Eulerian-Lagrangian analysis for particle velocities and trajectories in a pure wave motion using particle image velocimetry, Philos. Trans. R. Soc. A Math. Phys. Eng. Sci. 370(1964) (2014) 1687-1702.

[6] A. D. Craik, The origins of water wave theory, Annu. Rev. Fluid Mech. 36(2004) 1-28.

[7] D. Henry, The Trajectories of Particles in Deep-Water Stokes Waves, Int. Math. Res. Not. 2006(Article ID 23405) (2006) 1-13.

[8] A. Constantin and W. Strauss, Pressure beneath a Stokes wave, Commun. Pure Appl. Math. 63 (2010) 533-557.

[9] A. Constantin and G. Villari, Particle Trajectories in Linear Water Waves, J. Math. Fluid Mech. 10(2008) 1-18.

[10] A. Constantin, M. Ehrnström and G. Villari, Particle trajectories in linear deep-water waves, Nonlinear Anal. Real World Appl. 9(2008) 1336-1344.

[11] H. Okamoto and M. Shoji, Trajectories of fluid particles in a periodic water wave., Philos. Trans. A. Math. Phys. Eng. Sci. 370(April 2012) 1661-76.

[12] R. S. Johnson, Edge waves: theories past and present., Philos. Trans. Roy. Soc. London Ser. A 365(September 2007) 2359-76.

[13] G. B. Whitham, Lectures on Wave Propagation, Tata Institute of Fundamental Research (Bombay, 1979).

[14] U. T. Ehrenmark, A note on tuning in Roseau's alternative edge waves, J. Fluid Mech. 382 (1999) 245-262.

[15] A. Constantin, Edge waves along a sloping beach, J. Phys. A. Math. Gen. 34(November 2001) 97239731.

[16] H. Yeh, A Note on Edge Waves, in Coast. Hydrodyn., ed. R. A. Dalrymple (American Society of Civil Engineers, 1987), pp. 256-269.

[17] F. Ursell, Edge Waves on a Sloping Beach, Proc. R. Soc. A Math. Phys. Eng. Sci. 214(August 1952) 79-97.

[18] M. Roseau, Short waves parallel to the shore over a sloping beach, Comm. Pure Appl. Math. 11 (1958) 433-493.

[19] K. M. Mok and H. Yeh, On mass transport of progressive edge waves, Phys. Fluids 11(10) (1999) p. 2906.

[20] G. B. Whitham, Nonlinear effects in edge waves, J. Fluid Mech. 74 (1976) 353-368.

[21] J. V. Wehausen and E. V. Laitone, Surface Waves in Encyclopaedia of Physics, Vol. IX (Springer Verlag, 1960) 446-778.

[22] H. H. Yeh, Shoreline Profile of Stokes-Mode Edge Waves, J. Waterw. Port, Coastal, Ocean Eng. 118(1992) 112-116.

[23] M. Ehrnström, J. Escher and B.-V. Matioc, Two-dimensional steady edge waves. Part I: Periodic waves, Wave Motion 46(2009) 363-371.

[24] R. S. Johnson, Some contributions to the theory of edge waves, J. Fluid Mech. 524 (2005) 81-97. 